\title{
X-Ray Observations of GRS 1915+105
}

\section{J. Greiner}

Max-Planck-Institute for extraterrestrial Physics, 85740 Garching, FRG

\author{
B.A. Harmon, W.S. Paciesas
}

Marshall Space Flight Center, Huntsville, AL 35812, USA

E.H. Morgan, R.A. Remillard

Center for Space Research, MIT, Cambridge, MA 02139, USA

\section{Spectral analysis of simultaneous ROSAT/BATSE data}

After the discovery of GRS 1915+105 (Castro-Tirado et al. 1992) we obtained pointed ROSAT observations every six months (12 until now). The flux in the ROSAT $(0.1-2.4 \mathrm{keV})$ band is strikingly different from the simultaneous BATSE (25-50 keV) flux which was obtained by integrating the best fit power law (Fig. 1). Motivated by the different intensity evolution in the soft and hard X-ray band we have selected BATSE monitoring data collected simultaneously to ROSAT data and performed joint spectral fitting with XSPEC. As a result, we never got an acceptable fit (see Fig. 2): The BATSE power law (upper dotted line) is too steep to match the ROSAT band, and even allowing for an increased absorbing column (lower dotted line) does not solve the problem. Alternatively, neither a thermal bremsstrahlung fit (solid line) nor a power law fit (lower dash-dot line) to the ROSAT data match the BATSE flux. The upper dash-dot line is a -2.5 powerlaw which would match the BATSE data while giving too much 1-2 $\mathrm{keV}$ emission. A similar, but less stringent result is obtained when folding the best fit BATSE power law models with the HRI detector response to compare the expected count rate with the observed one. We therefore conclude that the spectrum during all simultaneous ROSAT/BATSE observations seemingly consists of two different spectral components.

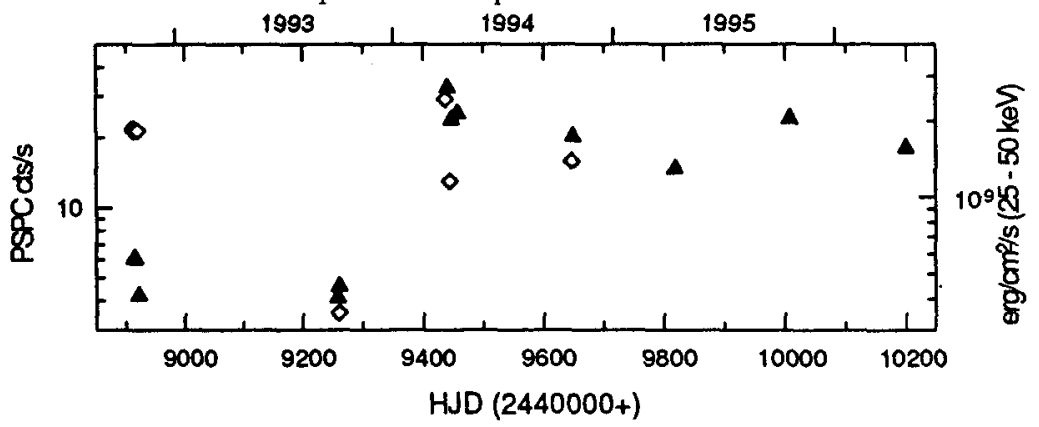

Figure 1. Intensities in the soft 0.1-2.4 keV ROSAT band (filled triangles, left axis) and the 25-50 keV BATSE band (open squares, right axis). 


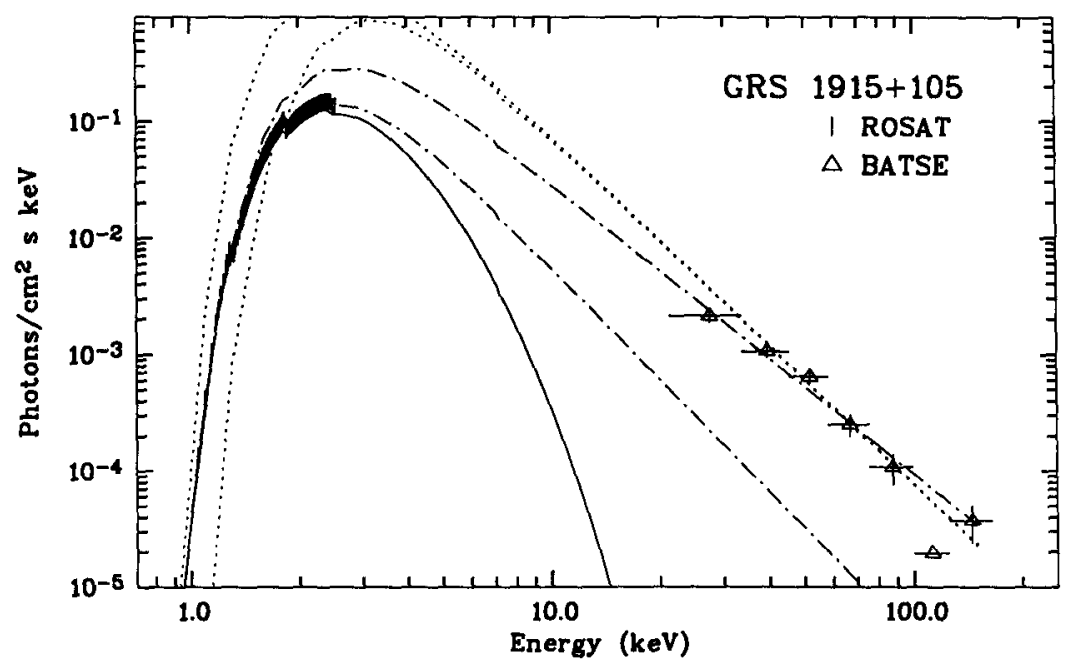

Figure 2. Spectral fits of simultaneous ROSAT/BATSE data of 19/20 October 1992 (see text). This demonstrates that none of the single component models satisfactorily fits the combined simultaneous data.

\section{Pointed RXTE Observations}

Immediately after the discovery of the high activity level of GRS 1915+105 in the RXTE ASM data it was observed in a series of pointings starting on April 6,1996 . Details of the drastic X-ray intensity changes on a variety of time scales ranging from sub-seconds to days are given elsewhere (Greiner, Morgan $\&$ Remillard 1996). Summarizing, the observations over a time span of 13 weeks reveal 3 episodes (April 6, May 20-26, June 11-19) of large amplitude variations between 0.2 and $3 \mathrm{Crab}$. In particular, the source exhibits quasi-periodic brightness sputters with varying duration and repetition time scale. In between these episodes GRS $1915+105$ remains at $\approx 1$ Crab with smaller amplitude variations, but higher frequency. The spectrum during the brightness sputters is remarkably different from the spectrum of the mean high state emission. We argue that such sputtering episodes are possibly caused by a major accretion disk instability. Based on the coincidence in time of two radio flares (Pooley 1996) following the observed X-ray sputtering episodes we speculate that superluminal ejections (as observed from GRS $1915+105$ during earlier activity periods) are related to episodes of large amplitude X-ray variations.

Acknowledgments. JG is supported by the German Bundesministerium für Bildung, Wissenschaft, Forschung und Technologie under contract No. FKZ 50 OR 9201. EHM and RR were supported by NASA contract NAS5-30612.

\section{References}

Castro-Tirado A.J., Brandt S., Lund N., 1992, IAU Circ. 5590

Greiner J., Morgan E.H., Remillard R.A., 1996, ApJ Lett. (in press)

Pooley G., 1996, IAU Circ. 6411 